\title{
Performance Analysis on Maximum Likelihood Detection for Two Input Multiple Output Systems
}

\author{
Wei Peng ${ }^{1}$, Shaodan $\mathrm{Ma}^{2}$, Tung-Sang $\mathrm{Ng}^{2}$, Jiangzhou $\mathrm{Wang}^{3}$ and Fumiyuki Adachi ${ }^{1}$ \\ ${ }^{1}$ Department of Electrical and Communication Engineering, Tohoku University, Japan \\ ${ }^{2}$ Department of Electrical and Electronic Engineering, the University of Hong Kong, Hong Kong \\ ${ }^{3}$ Department of Electronics, the University of Kent, UK
}

\begin{abstract}
This paper addresses the problem of performance analysis for maximum likelihood (ML) detection in two-input multiple-output multiplexing systems. A novel analytical method is presented to formulate the symbol error probability (SEP). Based on the total probability theory, the SEPs of the two transmitted signals are obtained in closed-form by solving the SEP equations. Both equal and unequal power allocations are investigated. The accuracy of the proposed method is verified by Monte-Carlo simulations. The proposed method can also be extended to systems with more than two inputs.
\end{abstract}

Index terms: MIMO Multiplexing, Symbol Error Probability, Maximum Likelihood.

\section{INTRODUCTION}

Multiple-input multiple-output (MIMO) multiplexing has been regarded as one of the most significant techniques to improve the system capacity in recent years [1]. A number of detection algorithms have been proposed, e.g., zero forcing $(\mathrm{ZF})$, minimum mean squared error (MMSE) [2], vertical Bell laboratories space time (V-BLAST) [3] and maximum likelihood (ML) [4] algorithms. Among them, ML detection is the optimal one from the error probability point of view. Since ML is a non-linear detection algorithm, performance analysis which is instructive for system designers is not straight-forward. In the literature, the symbol error probability (SEP) or bit error rate (BER) is generally evaluated as a union bound based on the calculation of pair-wise error probabilities (PEP) where PEP means the probability that the receiver decides in favor of one signal vector when another signal vector is transmitted. Upper-bound/approximation of the SEP or BER is then derived based on the PEP expressions [4-9]. Unfortunately, all of these analytical bounds/ approximations are tight only under high signal-to-noise-ratio (SNR) and there is a significant gap between the analytical and simulation results when the SNR is low.

In this paper, a novel SEP analysis method for the ML detection in a MIMO multiplexing system with two transmit antennas is proposed. In this method, the SEP for one transmitted signal is expressed in terms of the SEPs conditioned on the error of the other transmitted signal. By analyzing the post-detection-SNR and developing the conditional SEPs, the SEPs are finally obtained in closed-form by solving the SEP equations. Unlike the existing works [4-9] where equal power allocation is assumed, unequal power allocation between the transmitted signals is also considered. Since unequal power allocation is generally the case in many practical systems, e.g., beamforming systems. The proposed method is more practical than the existing ones. The accuracy of this SEP analysis is demonstrated by Monte-Carlo simulations. The comparisons between the analytical and simulation results show that they match quite well even under low SNR situation. In addition, the proposed method can also be extended to the systems with more than two inputs.

The rest of the paper is organized as follows. Section II introduces the MIMO multiplexing system model and the ML detection. The proposed analysis method is presented in Section III. In Section IV, the accuracy of the proposed method is investigated by Monte-Carlo simulations. Finally, conclusions are drawn in Section V.

\section{SYSTEM MODEL AND ML DETECTION}

\section{A. System model}

Consider a $2 \times N \quad$ MIMO multiplexing system with 2 transmit and $N$ receive antennas $(N \geq 2)$. The baseband received signal vector is given by

$$
\mathbf{y}=\mathbf{H} \mathbf{x}+\mathbf{n}
$$

where $\mathbf{y}=\left[y_{1}, \cdots, y_{N}\right]^{T}$ is an $N \times 1$ vector with $y_{j}$ being the received signal at the $j^{\text {th }}$ receive antenna; superscript $T$ represents matrix transpose; $\mathbf{n}=\left[n_{1}, \cdots, n_{N}\right]^{T}$ is an $N \times 1$ additive complex Gaussian noise (AWGN) vector, each element being independent with zero mean and variance $\sigma_{n}^{2} ; \mathbf{H}$ is an $N \times 2$ channel matrix whose $(j, i)^{\text {th }}$ element $h_{j, i}$ stands for the channel gain from the $i^{\text {th }}$ transmit antenna $(i=1,2)$ to the $j^{\text {th }}$ receive antenna $(j=1, \cdots, N)$ and is assumed to be an independently and identically distributed (i.i.d) complex Gaussian variable with zero mean and unit variance $\left(\sigma_{h}^{2}=1\right) ; \quad \mathbf{x}=\left[x_{1}, x_{2}\right]^{T}$ is a $2 \times 1$ vector with the $i^{\text {th }}(i=1,2)$ element being the transmitted signal from the $i^{\text {th }}$ transmit antenna and independent from the other elements and the noises. Let $C$ represent the constellation of the transmitted signals. It is assumed that all the symbols in the constellation have equal probability. To simplify the 
derivation, the transmitted signals are assumed to be quadrature phase shift keying (QPSK) modulated. The proposed method can be easily extended to systems using other modulation schemes.

\section{B. Maximum Likelihood detection}

When the noise is Gaussian distributed, ML detection for the transmitted signals can be realized as [10]

$$
\tilde{\mathbf{x}}=\underset{\tilde{\mathbf{x}}}{\arg \min } \sum_{j=1}^{N}\left|y_{j}-\sum_{i=1}^{2} h_{j, i} \tilde{x}_{i}\right|^{2},
$$

where $\tilde{\mathbf{x}}$ represents the decision vector for $\mathbf{x}$ and $\tilde{x}_{i}$ represents the decision for $x_{i}$.

\section{Symbol ERror Probability}

The SEP of $x_{i}$ can be written in terms of the SEPs conditioned on the error of $x_{\bar{i}}$ and the SEP of $x_{\bar{i}}$ [11] as

$$
\begin{aligned}
P\left(\tilde{x}_{i} \neq x_{i}\right)= & P\left(\tilde{x}_{i} \neq x_{i} \mid \tilde{x}_{\bar{i}}=x_{\bar{i}}\right)\left[1-P\left(\tilde{x}_{\bar{i}} \neq x_{\bar{i}}\right)\right] . \\
& +P\left(\tilde{x}_{i} \neq x_{i} \mid \tilde{x}_{\bar{i}} \neq x_{\bar{i}}\right) P\left(\tilde{x}_{\bar{i}} \neq x_{\bar{i}}\right)
\end{aligned}
$$

where $\bar{i}$ is used to denote the index of the signal transmitted from the other antenna; $P\left(\tilde{x}_{i} \neq x_{i} \mid \tilde{x}_{\bar{i}}=x_{\bar{i}}\right)$ denotes the SEP of $x_{i}$ conditioned on the event that the decision for $x_{\bar{i}}$ is correct; while $P\left(\tilde{x}_{i} \neq x_{i} \mid \tilde{x}_{\bar{i}} \neq x_{\bar{i}}\right)$ stands for the SEP of $x_{i}$ conditioned on the event that the decision for $x_{\bar{i}}$ is wrong.

\section{A. Conditional SEP P $P\left(\tilde{x}_{i} \neq x_{i} \mid \tilde{x}_{\bar{i}}=x_{\bar{i}}\right)$}

The conditional SEP $P\left(\tilde{x}_{i} \neq x_{i} \mid \tilde{x}_{\bar{i}}=x_{\bar{i}}\right)$ is analyzed first. The result in this sub-section will also form the basis for the following analysis on the conditional SEP $P\left(\tilde{x}_{i} \neq x_{i} \mid \tilde{x}_{\bar{i}} \neq x_{\bar{i}}\right)$. When $\tilde{x}_{\bar{i}}=x_{\bar{i}}$, it follows from (2) that the detection of $x_{i}$ becomes

$$
\tilde{x}_{i}=\underset{\tilde{x}_{i}}{\arg \min } \sum_{j=1}^{N}\left|h_{j, i} x_{i}+n_{j}-h_{j, i} \tilde{x}_{i}\right|^{2} .
$$

In fact, the detection of $x_{i}$ in (4) is equivalent to the ML detection in a single-input multiple-output (SIMO) system where the transmitted signal $x_{i}$ experiences $N$ independent fading channels $h_{j, i}(1 \leq j \leq N)$ and is corrupted by $N$ additive noises $n_{j}$. Thus the result for a SIMO system in [12] can be applied here and the post-detection-SNR is given as

$$
\begin{aligned}
\gamma_{i, \tilde{x}_{\bar{i}}=x_{\bar{i}}} & =\sum_{j=1}^{N} \gamma_{j, i, \tilde{x}_{i}=x_{\bar{\tau}}} \\
& =\sum_{j=1}^{N}\left|h_{j, i}\right|^{2}\left|x_{i}\right|^{2} / \sigma_{n}^{2}=\omega_{i}\left|x_{i}\right|^{2} / \sigma_{n}^{2},
\end{aligned}
$$

where $\gamma_{j, i, \tilde{x}_{\bar{T}}=x_{\bar{\tau}}}=\left|h_{j, i}\right|^{2}\left|x_{i}\right|^{2} / \sigma_{k^{2}}^{2}$ is the SNR on the $j^{\text {th }}$ fading channel and $\omega_{i}=\sum_{j=1}^{N^{\mu}}\left|h_{j, i}\right|^{2}$. It follows that for QPSK modulated systems, the SEP conditioned on $x_{i}, \omega_{i}$ and $\tilde{x}_{\bar{i}}=x_{\bar{i}}$ can be written as [13]

$$
\begin{aligned}
& p\left(\tilde{x}_{i} \neq x_{i} \mid x_{i}, \omega_{i}, \tilde{x}_{\bar{i}}=x_{\bar{i}}\right)=G\left(\gamma_{i, \tilde{x}_{\bar{i}}=x_{\bar{i}}}\right), \\
& =2 Q\left(\sqrt{\gamma_{i, \tilde{x}_{\bar{i}}=x_{\bar{i}}}}\right)-Q^{2}\left(\sqrt{\gamma_{i, \tilde{x}_{\bar{i}}=x_{\bar{i}}}}\right),
\end{aligned}
$$

where $Q(t)=\int^{\infty} 1 / \sqrt{2 \pi} \cdot \exp \left(-z^{2} / 2\right) d z$. It is observed in [14] that ${ }^{\pi / 2} Q(t)$ can be rewritten as $Q(t)=1 / \pi \cdot \int_{0}^{\pi / 2} \exp \left(-t^{2} / 2 \sin ^{2} \theta\right) d \theta$ and therefore the conditional SEP in (6) is equivalent to

$$
\begin{aligned}
& p\left(\tilde{x}_{i} \neq x_{i} \mid x_{i}, \omega_{i}, \tilde{x}_{\bar{i}}=x_{\bar{i}}\right) \\
= & \frac{1}{\pi} \int_{0}^{3 \pi / 4} \exp \left(-\frac{\gamma_{i, \tilde{x}_{\bar{T}}=x_{\bar{i}}}}{2 \sin ^{2} \theta}\right) d \theta . \\
= & \frac{1}{\pi} \int_{0}^{3 \pi / 4} \exp \left(-\frac{\omega_{i}\left|x_{i}\right|^{2} / \sigma_{n}^{2}}{2 \sin ^{2} \theta}\right) d \theta
\end{aligned}
$$

It should be noted that the function $G(\cdot)$ in (6) depends on the modulation scheme. It is straight-forward to apply this method to the systems using other modulations by altering $G(\cdot)$. By averaging (7) with respect to the statistics of $x_{i}$ and $\omega_{i}$, the average conditional SEP can be achieved as

$$
\begin{aligned}
& P\left(\tilde{x}_{i} \neq x_{i} \mid \tilde{x}_{\bar{i}}=x_{\bar{i}}\right) \\
= & \sum_{x_{i} \in C} p\left(x_{i}\right) \int_{0}^{\infty} p\left(\tilde{x}_{i} \neq x_{i} \mid x_{i}, \omega_{i}, \tilde{x}_{\bar{i}}=x_{\bar{i}}\right) p\left(\omega_{i}\right) d \omega_{i}, \\
= & \sum_{x_{i} \in C} p\left(x_{i}\right) \frac{1}{\pi} \int_{0}^{3 \pi / 4} \int_{0}^{\infty} \exp \left(-\frac{\omega_{i}\left|x_{i}\right|^{2}}{2 \sigma_{n}^{2} \sin ^{2} \theta}\right) p\left(\omega_{i}\right) d\left(\omega_{i}\right) d \theta
\end{aligned}
$$

where $p\left(x_{i}\right)$ and $p\left(\omega_{i}\right)$ are the probability density function (p.d.f) of $x_{i}$ and $\omega_{i}$, respectively. Apparently from the definition of $\omega_{i}$, it is a chi-square distributed variable [13] with $2 N$ degrees of freedom. It follows that the p.d.f of $\omega_{i}$ is given by

$$
p\left(\omega_{i}\right)=\frac{\omega_{i}^{N-1} \exp \left(-\omega_{i}\right)}{(N-1) !},
$$

Substituting (9) into (8), the conditional SEP becomes 


$$
\begin{aligned}
& P\left(\tilde{x}_{i} \neq x_{i} \mid \tilde{x}_{\bar{i}}=x_{\bar{i}}\right) \\
= & \frac{1}{\pi \Gamma(N)} \sum_{x_{i} \in C} p\left(x_{i}\right) \int_{0}^{3 \pi / 4} \int_{0}^{\infty} \omega_{i}^{N-1} \exp \left[-\omega_{i}\left(1+\frac{\left|x_{i}\right|^{2}}{2 \sigma_{n}^{2} \sin ^{2} \theta}\right)\right] d \omega_{i} d \theta . \\
= & \frac{1}{\pi} \sum_{x_{i} \in C} p\left(x_{i}\right) \int_{0}^{3 \pi / 4}\left(1+\frac{\left|x_{i}\right|^{2}}{2 \sigma_{n}^{2} \sin ^{2} \theta}\right)^{-N} d \theta
\end{aligned}
$$

Note that $\int_{0}^{\infty} x^{n} \exp (-\mu x) d x=n ! \mu^{-n-1} \quad$ [15] is used in the above derivation.

\section{B. Conditional SEP $P\left(\tilde{x}_{i} \neq x_{i} \mid \tilde{x}_{\bar{i}} \neq x_{\bar{i}}\right)$}

When $\tilde{x}_{\bar{i}} \neq x_{\bar{i}}$, the received signal vector can be rewritten as

$$
\mathbf{y}=\mathbf{h}_{i} x_{i}+\mathbf{h}_{\bar{i}} \tilde{x}_{\bar{i}}+\mathbf{h}_{\bar{i}} \Delta x_{\bar{i}}+\mathbf{n},
$$

where $\mathbf{h}_{i}$ is the $i^{\text {th }}$ column of the channel matrix $\mathbf{H}$ and $\Delta x_{\bar{i}}=x_{\bar{i}}-\tilde{x}_{\bar{i}}$. This situation can be regarded as if $\tilde{x}_{\bar{i}}$ were transmitted from the $\bar{i}^{\text {th }}$ antenna and $\mathbf{h}_{\bar{i}} \Delta x_{\bar{i}}$ in (11) will then be treated as interference. As a result, $\mathbf{h}_{\bar{i}} \Delta x_{\bar{i}}+\mathbf{n}$ is considered as the equivalent noise vector and the received signal vector is expressed as

$$
\mathbf{y}=\mathbf{h}_{i} x_{i}+\mathbf{h}_{\bar{i}} \tilde{x}_{\bar{i}}+\mathbf{v}_{\bar{i}}
$$

where $\mathbf{v}_{\bar{i}}=\mathbf{h}_{\bar{i}} \Delta x_{\bar{i}}+\mathbf{n}$ denotes the equivalent noise vector with its $j^{\text {th }}(j=1,2, \cdots, N)$ element given by $v_{\bar{i}, j}=h_{j, \bar{i}} \Delta x_{\bar{i}}+n_{j}$. Since $h_{j, \bar{i}}$ and $n_{j}$ are independent zero mean complex Gaussian variables with variance one and $\sigma_{n}^{2}$ respectively, it follows that for given $\mathbf{x}$ and $\Delta x_{\bar{i}}, \quad v_{\bar{i}, j}$ is also complex Gaussian variable with zero mean and variance given as [13]

$$
\sigma_{v, \bar{i}}^{2}=\operatorname{var}\left\{h_{j, \bar{i}} \Delta x_{\bar{i}}+n_{j}\right\}=\left|\Delta x_{\bar{i}}\right|^{2}+\sigma_{n}^{2} .
$$

As the symbol error occurs in adjacent positions in the constellation with the highest probability, it is reasonable to assume that error only happens between the transmitted symbol and its nearest constellation neighbor. Under this $\left|\Delta x_{\bar{i}}\right|^{2} \approx \min \left(d_{\bar{i}, c}^{2}\right)=\alpha_{\bar{i}} E\left\{\left|x_{\bar{i}}\right|^{2}\right\} \quad$ be approximated as represents the minimum square Euclidean distance (SED) between $x_{\bar{i}}$ and its constellation neighbors, $E\left\{\left|x_{\bar{i}}\right|^{2}\right\}$ is the average transmit power of $x_{\bar{i}}$ and $\alpha_{\bar{i}}$ stands for the ratio of $\min \left(d_{\bar{i}, c}^{2}\right)$ to the average transmit power of $x_{\bar{i}}$. Note that $\min \left(d_{\bar{i}, c}^{2}\right)$ and $\alpha_{\bar{i}}$ vary with the modulation scheme. For QPSK modulation, the minimum SED between the correct symbol and its nearest neighbor is as twice as the average symbol energy, i.e., $\alpha_{\bar{i}}=2 E\left\{\left|x_{\bar{i}}\right|^{2}\right\}$ and $\alpha_{\bar{i}}=2$.

Now the average SEP conditioned on $\tilde{x}_{\bar{i}} \neq x_{\bar{i}}$ can be analyzed in the same way as in sub-Section III- $A$. From (2) and (12), the detection of $x_{i}$ satisfies

$$
\tilde{x}_{i}=\underset{\tilde{x}_{i}}{\arg \min } \sum_{j=1}^{N}\left|h_{j, i} x_{i}+v_{\bar{i}, j}-h_{j, i} \tilde{x}_{i}\right|^{2} .
$$

Equation (14) also represents the ML detection for a SIMO system and the post-detection-SNR under condition $\tilde{x}_{\bar{i}} \neq x_{\bar{i}}$ becomes

$$
\begin{aligned}
& \gamma_{i, \tilde{x}_{\bar{i}} \neq x_{\bar{i}}}=\sum_{j=1}^{N} \gamma_{j, i, \tilde{x}_{\bar{i}} \neq x_{\bar{i}}} \\
& =\sum_{j=1}^{N}\left|h_{j, i}\right|^{2}\left|x_{i}\right|^{2} / \sigma_{v, \bar{i}}^{2}=\omega_{i}\left|x_{i}\right|^{2} / \sigma_{v, \bar{i}}^{2} .
\end{aligned}
$$

It follows that the conditional SEP $p\left(\tilde{x}_{i} \neq x_{i} \mid x_{i}, \omega_{i}, \tilde{x}_{\bar{i}} \neq x_{\bar{i}}\right)$ is similar to (7) with $\gamma_{i, \tilde{x}_{\bar{i}}=x_{\bar{i}}}$ replaced by $\gamma_{i, \tilde{x}_{\bar{i}} \neq x_{\bar{i}}}$ as

$$
\begin{aligned}
& p\left(\tilde{x}_{i} \neq x_{i} \mid x_{i}, \omega_{i}, \tilde{x}_{\bar{i}} \neq x_{\bar{i}}\right)=G\left(\gamma_{i, \tilde{x}_{\bar{i}} \neq x_{\bar{i}}}\right) \\
& =\frac{1}{\pi} \int_{0}^{3 \pi / 4} \exp \left(-\frac{\omega_{i}\left|x_{i}\right|^{2} / \sigma_{v, \bar{i}}^{2}}{2 \sin ^{2} \theta}\right) d \theta
\end{aligned}
$$

The average conditional SEP $P\left(\tilde{x}_{i} \neq x_{i} \mid \tilde{x}_{\bar{i}} \neq x_{\bar{i}}\right)$ is then obtained similarly to (10) with $\sigma_{n}^{2}$ replaced by $\sigma_{v, \bar{i}}^{2}$ as

$$
\begin{aligned}
& P\left(\tilde{x}_{i} \neq x_{i} \mid \tilde{x}_{\bar{i}} \neq x_{\bar{i}}\right) \\
& =\frac{1}{\pi} \sum_{x_{i} \in C} p\left(x_{i}\right) \int_{0}^{3 \pi / 4}\left(1+\frac{\left|x_{i}\right|^{2}}{2 \sigma_{v, \bar{i}}^{2} \sin ^{2} \theta}\right)^{-N} d \theta
\end{aligned}
$$

C. $P\left(\tilde{x}_{1} \neq x_{1}\right)$ and $P\left(\tilde{x}_{2} \neq x_{2}\right)$

Applying the conditional SEPs (10) and (17) into (3), two SEP equations concerning $x_{1}$ and $x_{2}$ will be generated. Thus, the SEPs $P\left(\tilde{x}_{i} \neq x_{i}\right),(i=1,2)$ can be obtained in closed-form by solving the SEP equations. Generally, the power allocation will affect the SEPs. In the following, we will derive the SEPs under both equal and unequal power allocations.

\section{C.1 Equal power allocation}

When equal power is allocated, the SEPs of the two transmitted signals are the same, that is $P\left(\tilde{x}_{1} \neq x_{1}\right)=P\left(\tilde{x}_{2} \neq x_{2}\right)=e$. It follows from (3), (10) and (17) that the SEP equation is given by 


$$
\begin{aligned}
e & =P\left(\tilde{x}_{i} \neq x_{i} \mid \tilde{x}_{\bar{i}}=x_{\bar{i}}\right)(1-e) \\
& +P\left(\tilde{x}_{i} \neq x_{i} \mid \tilde{x}_{\bar{i}} \neq x_{\bar{i}}\right) e
\end{aligned}
$$

The closed-form SEP is thus the solution of (18) given as

$$
\begin{aligned}
& P\left(\tilde{x}_{1} \neq x_{1}\right)=P\left(\tilde{x}_{2} \neq x_{2}\right)=e \\
& =\frac{P\left(\tilde{x}_{i} \neq x_{i} \mid \tilde{x}_{\bar{i}}=x_{\bar{i}}\right)}{1-\left[P\left(\tilde{x}_{i} \neq x_{i} \mid \tilde{x}_{\bar{i}} \neq x_{\bar{i}}\right)-P\left(\tilde{x}_{i} \neq x_{i} \mid \tilde{x}_{\bar{i}}=x_{\bar{i}}\right)\right]} .
\end{aligned}
$$

\section{C.2 Unequal power allocation}

When unequal power is allocated, the SEPs of the two transmitted signals will be different. Let $P\left(\tilde{x}_{1} \neq x_{1}\right)=\varepsilon_{1}$ and $P\left(\tilde{x}_{2} \neq x_{2}\right)=\varepsilon_{2}$, where $\varepsilon_{1} \neq \varepsilon_{2}$. In this situation, two SEP equations are obtained from (3), (10) and (17) as

$$
\left\{\begin{array}{rl}
\varepsilon_{1}= & P\left(\tilde{x}_{1} \neq x_{1}\right)=P\left(\tilde{x}_{1} \neq x_{1} \mid \tilde{x}_{2}=x_{2}\right)\left(1-\varepsilon_{2}\right) \\
& +P\left(\tilde{x}_{1} \neq x_{1} \mid \tilde{x}_{2} \neq x_{2}\right) \varepsilon_{2} \\
\varepsilon_{2} & =P\left(\tilde{x}_{2} \neq x_{2}\right)=P\left(\tilde{x}_{2} \neq x_{2} \mid \tilde{x}_{1}=x_{1}\right)\left(1-\varepsilon_{1}\right) \\
& +P\left(\tilde{x}_{2} \neq x_{2} \mid \tilde{x}_{1} \neq x_{1}\right) \varepsilon_{1}
\end{array} .\right.
$$

By solving (20), the SEPs are obtained in closed-form as

$$
\left\{\begin{array}{c}
\varepsilon_{1}=P\left(\tilde{x}_{1} \neq x_{1}\right)=\frac{\lambda_{0}+\left(\lambda_{1}-\lambda_{0}\right) \beta_{0}}{1-\left(\lambda_{1}-\lambda_{0}\right)\left(\beta_{1}-\beta_{0}\right)} \\
\varepsilon_{2}=P\left(\tilde{x}_{2} \neq x_{2}\right)=\frac{\beta_{0}+\left(\beta_{1}-\beta_{0}\right) \lambda_{0}}{1-\left(\lambda_{1}-\lambda_{0}\right)\left(\beta_{1}-\beta_{0}\right)}
\end{array}\right.
$$

where $\left\{\begin{array}{l}\lambda_{0}=P\left(\tilde{x}_{1} \neq x_{1} \mid \tilde{x}_{2}=x_{2}\right) \\ \lambda_{1}=P\left(\tilde{x}_{1} \neq x_{1} \mid \tilde{x}_{2} \neq x_{2}\right)\end{array}\right.$ and

$$
\left\{\begin{array}{l}
\beta_{0}=P\left(\tilde{x}_{2} \neq x_{2} \mid \tilde{x}_{1}=x_{1}\right) \\
\beta_{1}=P\left(\tilde{x}_{2} \neq x_{2} \mid \tilde{x}_{1} \neq x_{1}\right)
\end{array} .\right.
$$

\section{Extension to systems with more than two inputs}

When the systems with more than two inputs are considered, the proposed method can still be applied to obtain the SEPs. To do this, the SEP equations will be set up by expanding the SEPs of the transmitted signals according to total probability theory [16]. The conditional SEPs and the probabilities of the error events should be defined and evaluated according to the number of transmit antennas. Note that when the number of inputs is larger than three, the SEP equations may appear as polynomial equations and the solutions can be obtained with the aid of computational tools such as "fsolve" in Matlab.

\section{NUMERICAL AND SIMULATION RESUlT}

In the following examples, the number of receive antennas $N$ is chosen as 2 and 4 respectively. The channel gain between each pair of transmit and receive antennas is randomly generated complex Gaussian variable with zero mean and unit variance. The results are given with respect to the ratio of the average transmit power to the average noise power, $S N R=1 / \sigma_{n}^{2}$. The simulation results are obtained by averaging over $10^{6}$ Monte Carlo realizations.

\section{A. Equal power allocation}

It is assumed that $E\left\{\left|x_{1}\right|^{2}\right\}=E\left\{\left|x_{2}\right|^{2}\right\}=1$. The comparison between the analytical results obtained by (19) and the simulation results is shown in Fig. 1. It is obvious that the analytical results coincide with the simulation ones in the considered SNR region.

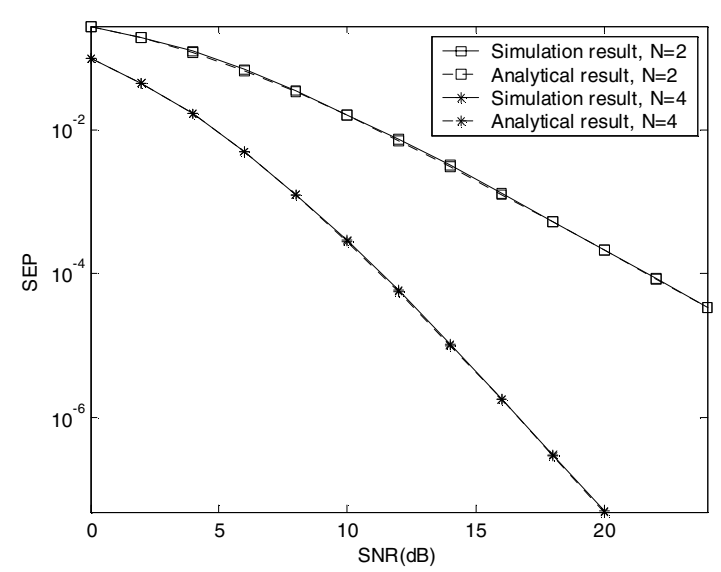

Fig.1 Results of $2 \times N$ systems, equal power allocation.

\section{B. Unequal power allocation}

It is assumed that $E\left\{\left|x_{1}\right|^{2}\right\}=7 / 4$ and $E\left\{\left|x_{2}\right|^{2}\right\}=1 / 4$. The analytical SEPs of $x_{1}$ and $x_{2}$ obtained from (21) are compared with the simulation ones. The results for the cases where $N=2$ and $N=4$ are shown in Fig. 2 and Fig. 3 respectively. Clearly, the analytical results match quite well with the simulation results in the considered SNR region. 


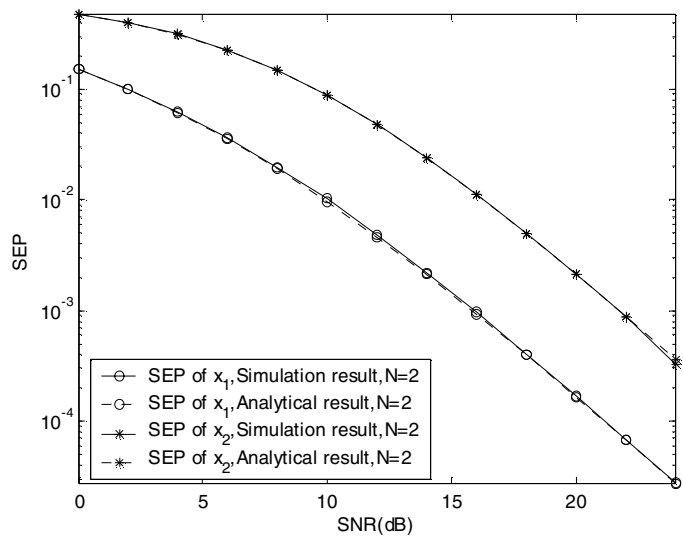

Fig.2 Results of a $2 \times 2$ system, unequal power allocations.

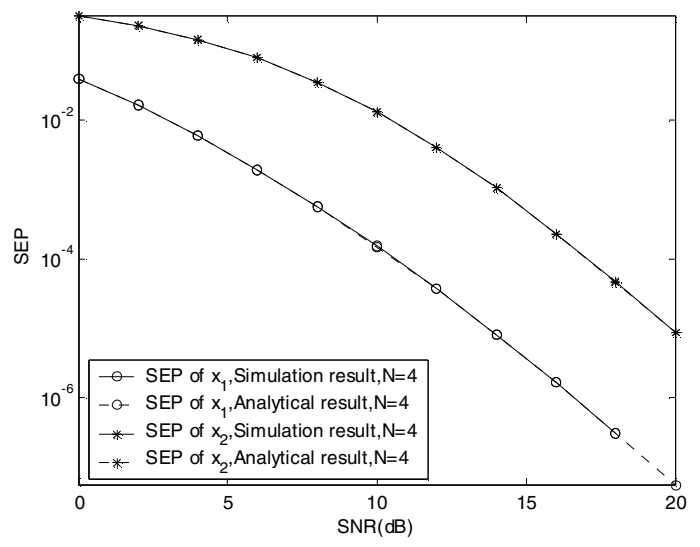

Fig.3 Results of a $2 \times 4$ system, unequal power allocation.

The comparisons between the analytical and simulation results demonstrate the accuracy of the proposed method under the situations of equal power allocation as well as unequal power allocation. This method provides system designers with an effective method to predict the system performance, even when the SNR is low. Note that in most existing methods [4-9], there is a significant gap between the analytical and simulation results in low SNR situations.

\section{CONCLUSIONS}

This paper has presented a novel analytical method to SEP analysis for ML detection in MIMO multiplexing systems with two transmit antennas. The SEP equations have been generated after analyzing the post-detection-SNR and deriving the conditional SEPs. The closed-form SEPs have been obtained by solving the SEP equations. Both equal and unequal power allocations are considered. Monte-Carlo simulations have demonstrated that the proposed method yield accurate results, even under low SNR. The proposed method can also be applied to systems with more than two inputs.

\section{ACKNOWLEDGEMENT}

This work was supported by the Hong Kong research grants council (Grant No.:7160/06E) and also partly supported by the Global COE program of the Tohoku University.

\section{REFERENCES}

[1] G. J. Foschini and M. J. Gans, "On limits of wireless communications in a fading environment when using multiple antennas," Wireless Personal Communications, vol. 6, pp. 311-335, March 1998.

[2] J. M. Wang and Daneshrad. B, "A comparative study of MIMO detection algorithms for wideband spatial multiplexing systems", IEEE conference on wireless communications and networking, vol. 1, pp408413, March 2005.

[3] G. D. Golden, G. J. Foschini, R. A. Valenzuela, and P. W. Wolniansky, "Detection Algorithm and Initial Laboratory Results Using V-BLAST Space-Time Communication Architecture,” IEEE Electronics Letters, Vol. 35, No. 1, January 1999.

[4] R. Van Nee, A. Van Zelst, and G. Awater, "Maximum likelihood decoding in a space division multiplexing system”, IEEE VTC 2000, vol.1, pp6-10, May 2000.

[5] M. Shin, D. S. Kwon and C. Lee, "Performance Analysis of Maximum Likelihood Detection for MIMO Systems", IEEE $63^{\text {rd }}$ Vehicular Technology Conference, 2006-Spring.

[6] G. Lindell, "Some exact union bound results for maximum likelihood detection in MIMO systems," IEEE International Symposium on Information Theory, pp. 2223 - 2227, September 2005.

[7] M. Kiessling, J. Speidel, N. Geng, and M. Reinhardt, "Performance analysis of MIMO maximum likelihood receivers with channel correlation, colored Gaussian noise, and linear pre-filtering," IEEE International Conference on Communications, vol. 5, vol.5, pp. 3026 3030, May 2003.

[8] H. T. Liu, G. Li, X. Q. Cheng and D. B. Li, "Performance Analysis Framework of ML MIMO Receiver over Correlated Rayleigh Fading Channel," 2006 IEEE International Conference on Communications, pp. 1634 - 1639 , May 2006.

[9] X. Zhu and R. D. Murch, "Performance analysis of maximum likelihood detection in a MIMO antenna system," IEEE Transactions on Communications, vol. 50, pp. 187-191, February 2002.

[10] M. Kay Steven, "Fundamentals of Statistical Signal Processing," Prentice Hall, 1993.

[11] Henry, S. John and W. Woods, "Probability and random processed with applications to signal processing", Prentice Hall, 2002.

[12] G. L. Stuber, "Principles of Mobile Communication," Kluwer Academic Publishers, 2000.

[13] J.G. Proakis, "Digital Communications", McGraw Hill, $4^{\text {th }}$ edition, 2001.

[14] M. K. Simon and M. S. Alouini, "Digital Communication over Fading Channels,” John Wiley \& Sons Inc., 2000.

[15] I. N. Bronshtein, K. A. Semendyayev, G. Musiol and H. Muehlig, "Handbook of mathematics," Springer, 1997.

[16] A. Papoulis, "Probability, Random Variables, and Stochastic Processes," McGraw-Hill, 1965. 